Rapport - Société canadienne d'histoire de l'Église catholique RAPPORT

\title{
Monseigneur Camille Roy : témoin d'une époque littéraire
}

\section{Jean Éthier Blais}

Volume 26, 1959

URI : https://id.erudit.org/iderudit/1007414ar

DOI : https://doi.org/10.7202/1007414ar

Aller au sommaire du numéro

Éditeur(s)

La Société canadienne d'histoire de l'Église catholique

ISSN

0318-6148 (imprimé)

1927-7075 (numérique)

Découvrir la revue

Citer cet article

Éthier Blais, J. (1959). Monseigneur Camille Roy : témoin d'une époque

littéraire. Rapport - Société canadienne d'histoire de l'Église catholique, 26,

51-56. https://doi.org/10.7202/1007414ar

Tous droits réservés @ La Société canadienne d'histoire de l'Église catholique, 1960
Ce document est protégé par la loi sur le droit d'auteur. L'utilisation des services d’Érudit (y compris la reproduction) est assujettie à sa politique d'utilisation que vous pouvez consulter en ligne.

https://apropos.erudit.org/fr/usagers/politique-dutilisation/ 


\title{
Monseigneur Camille Roy: Témoin d'une époque littéraire
}

\author{
(dédié à l'abbé Raymond Tremblay)
}

Le but que nous nous proposons en tentant d'analyser l'œuvre de $\mathrm{M}^{\mathrm{gr}}$ Camille Roy, n'est pas l'exaltation d'un homme qui aurait refusé, les lui eût-on offertes, les proportions héroïques, ni, à travers lui, l'apologie d'une époque. Plutôt, nous essayerons de jauger la portée de l'effort accompli par les temps qui furent siens, le milieu intellectuel qu'il fréquentait et de découvrir la continuité sous-jacente qui relie

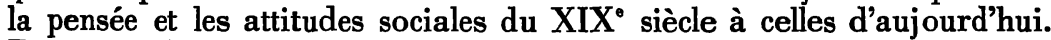
Eloignons donc, dès l'abord, l'idée d'un attachement à des détails précis, aux anecdotes historiques, à l'événement pour lui-même. Il nous a toujours paru que, dans l'histoire des idées d'une époque, la recherche des lignes de force intellectuelles devait primer. C'est dans cette perspective que nous nous placerons donc et tenterons de situer $\mathbf{M}^{\text {gr }}$ Camille Roy - témoin de son époque littéraire et par là même de son temps tout entier.

Témoin. Non pas que $\mathrm{M}^{\mathrm{gr}}$ Camille Roy témoigne entièrement de la vérité profonde que son temps portait en soi. Son rôle, plus limité, consiste à s'être penché, à travers le prisme de la littérature, sur les écrivains et à avoir, de cette sorte, soutenu et alimenté l'intérêt dans nos lettres, accru leur importance, créé le mode de notre critique. Mais voyons d'abord de plus près dans quelle époque la vie et l'œuvre de $\mathrm{M}^{\mathrm{gr}}$ Camille Roy vont s'insérer.

Camille Roy aura trente ans en 1900. Sans nous attacher aux luttes politiques qui, dans l'ensemble, ne lui ont jamais paru essentielles, voyons le milieu, le monde canadien-français où il s'insère. Il importe de noter tout d'abord que cette société, depuis cent trente ans, depuis que le traumatisme de la défaite l'a secouée d'outre en outre, n'est vouée qu'à l'épanouissement marginal. Issue de pauvres, maintenue dans la pauvreté, indifférente aux pouvoirs, craignant l'intelligence, elle n'a donné de grands esprits qu'aux partis politiques. En 1900, le Canada français n'avait produit ni un grand philosophe, ni un grand théologien, ni un grand homme, ni un saint. $\mathrm{Ni}$ par ailleurs, un grand industriel, un grand écrivain, un savant. La quête de la survivance avait tout pris. $M^{\text {gr }}$ Camille Roy se fera l'écho, en 1937, de l'esprit qui avait conduit à la situation de 1900. "Nous n'avons aucune répugnance ", écrirat-il, " nous, Canadiens français, à construire des manufactures et à conquérir la fortune. Mais nous croyons vraiment que ce n'est pas sur un monceau d'or ou sur un seuil d'usine que se trouve le point d'appui de notre influence canadienne-française en Amérique. » Il y a là une donnée fondamentale des penseurs, des 
théoriciens sociaux de l'époque; comme un rejet systématique de tout ce qui n'est pas esprit.

Par ailleurs, des courants contraires prennent leur essor. Au moment même où $\mathbf{M}^{\mathrm{gr}}$ Camille Roy débute dans la carrière des lettres, une classe nouvelle et importante commence d'apparaître dans notre vie nationale. Soucieuse d'acquérir les biens matériels, mais aussi, les biens de l'esprit : la science, les arts, les lettres. Cette montée de la classe moyenne s'accompagne de la recherche d'un équilibre et d'une continuité. $M^{\mathrm{gr}}$ Roy reprochera aux pères canadiens-français de ne pas se survivre dans leurs fils. Il constatait un passé; il n'analysait pas une tendance. Il semble aujourd'hui, avec le recul, qu'au contraire, le but même de cette nouvelle classe qui apparut au début de ce siècle fut, dès le premier jour, de consolider ses assises, de s'épanouir. Quelles formes prendront, si l'on analyse le peuple canadien-français dans son ensemble, cet affermissement des bases de la bourgeoisie canadiennefrançaise? Nous en voyons trois : a) la montée de la nouvelle vague nationaliste; b) l'acceptation positive de notre être national comme français et enfin, c) l'apparition et l'organisation des formes sociales de la liberté. Aux deux premières poussées de la bourgeoisie canadienne-française, $M^{\mathrm{gr}}$ Roy donna son appui entier. Un appui entier certes, mais nuancé. Après avoir, avec une sympathique attention parcouru son œuvre, nous en sommes venu à la conclusion que, dans le domaine des impondérables, il était passé maître. Et nulle part dans sa vie, cette recherche de la nuance ne s'est affirmée avec plus de sensibilité que dans son appui au nationalisme canadien-français et au fait français en Amérique. Voyons son attitude de plus près.

D'abord, il ne concevait pas l'un sans l'autre. Du fait français au Canada, de notre présence sur ce continent, devaient découler fatalement des prises de position de groupe - c'est-à-dire que l'acceptation réfléchie par le peuple canadien-français de sa qualité de français et de catholique devait l'entraîner, à un certain instant de son histoire sociale, à s'affirmer ce qu'il était. Par ailleurs, les forces contraires jouant, l'histoire passée, celle de la conquête et de l'appauvrissement progressif du peuple, les heurts de la politique, devaient presque fatatement donner à cette évolution le caractère primordial d'une lutte. C'est ce qu'un jeune écrivain et homme engagé d'aujourd'hui, M. JeanMarc Léger, appellera "une saine violence». Mais $\mathrm{M}^{\mathrm{gr}}$ Camille Roy par tempérament, par goût, par formation, n'était pas, ne pouvait être l'ami de cette violence, de ces écarts, sains ou pas. Son nationalisme n'était pas fondé en révolte - il était issu d'une connaissance profonde et d'un vivace amour de la civilisation française. Non pas que les travaux d'Hercule de l'abbé Groulx lui importassent peu. Au contraire. Mais il aspirait, son âme étant toute classique, à les situer dans le courant le plus intellectuel, et donc le moins soucieux de luttes, de la civilisation française. En sorte que, déplorant ce qu'il ne pouvait concevoir que comme un excès, se reposant sur l'histoire, il acceptait la lutte, il y participait même à sa façon - cette lutte étant à ses yeux plus la manifestation de la vitalité de l'esprit français en terre américaine, qu'un moyen de parvenir à des fins politiques. En un mot, au contraire de beaucoup de nationalistes de son époque, la 
pensée nationale de $\mathrm{M}^{\mathrm{gr}}$ Camille Roy était axée sur le fait français universel, sur la certitude qui était la sienne que les Canadiens français faisaient partie intégrante de la civilisation française, beaucoup plus que sur l'épanouissement d'une civilisation canadienne, issue de la civilisation française, mais différente d'elle. Peut-être tirerons-nous trop $\mathrm{M}^{\mathrm{gr}}$ Camille Roy dans la direction de nos pensées propres en disant que pour lui, l'histoire du Canada français n'était que l'histoire d'une nation civilisatrice, appelée France, malgré la brisure par l'océan, continuée ? Parlant de celle-ci, il disait : notre vieille histoire. Les événements auront apporté de l'eau à son moulin. Pour ne prendre qu'un exemple, l' "Union culturelle française ", peut-être à son insu, nous paraît issue des méditations de $\mathrm{M}^{\mathrm{gr}}$ Camille Roy sur notre passé et sur la continuité de ce passé français projetée jusque dans notre proche avenir.

Mais un autre mobile de l'acceptation totale par $\mathrm{M}^{\mathrm{gr}}$ Camille Roy du domaine intellectuel français, c'était sa condition de prêtre. Par elle, il se sentait doublement lié à notre passé. Parlant un jour de l'éducation au Canada, il faisait, avec une naturelle fierté, l'éloge de tous ces prêtres qui, du Petit Séminaire de Québec jusqu'aux Jésuites de Sudbury, avaient permis au peuple canadien-français de conserver son âme française, et par là, sa religion. Il n'eût rien abhorré de plus que la dissociation de ces deux forces vives, inséparables dans son esprit. Son œuvre, sa vie, ont proclamé l'indissolubilité de la langue française et de la religion catholique dans le contexte canadien-français. L'affermissement, aujourd'hui en passe de devenir réalité, de notre être national, lui eût-il rendu ces forces séparables? On nous permettra d'en douter. Son âme était proprement française; il n'y eût pas eu de problème et un manichéisme de cet ordre n'eût pas trouvé grâce à ses yeux.

Il est donc permis de concevoir $\mathrm{M}^{\mathrm{gr}}$ Camille Roy, devant la montée du fait national et ses virtualités nationalistes, comme l'homme, au sens strict et créateur, du juste milieu. En ceci, il représente une tendance profonde de l'intellectuel canadien et aura contribué à nourrir cette tendance d'une force positive. Il a situé la pensée nationale en dehors et au-dessus des considérations actuelles à son époque, considérations nées de la révolte contre l'injustice (donc d'une quête de la liberté) et infléchies presque automatiquement vers la violence à tout le moins verbale. Et en ceci, il est le témoin de tout un courant de notre pensée, qui ne rejette pas l'apport nationaliste, mais cherche à le canaliser dans le sens d'un épanouissement proprement français. Il y a une fierté d'être Canadien français. Mais selon que l'on appuie sur l'un ou l'autre des termes, tout en les maintenant unis, la direction de la pensée nationale est infléchie. La pensée nationale de $\mathrm{M}^{\mathrm{gr}}$ Camille Roy, c'est l'accent mis sur le mot "français ». Nous y voyons, pour nous, par-delà le régime français, les intendants et la chasse-galerie, un retour à nos sources authentiques, à notre véritable patrimoine national; nous faisons partie d'une lignée ininterrompue. Mais ces prémisses ne sont acceptables que si l'on accorde à l'esprit une primauté qui transcende les faits historiques. $\mathbf{M}^{\mathrm{gr}}$ Camille Roy croyait à cette primauté. Sa pensée, sans que peut-être il l'ait délibérément voulu, parvient donc jusqu'à nous : saine, pacifique, créatrice. 
Formé au Canada, puis à Paris, $\mathrm{M}^{\mathrm{gr}}$ Camille Roy est issu des disciplines classiques. Sa doctrine littéraire, appliquée à lui-même, reposait sur une ferveur un peu naïve devant les textes, l'ouverture de l'esprit, la primauté des formes littéraires raisonnables et la pureté de l'écriture. Faguet avait été son maître. Il lui reste fidèle. Dans l'étude des lettres, l'analyse des mobiles intérieurs, l'épanouissement des réflexes créateurs ne lui paraissent pas d'absolue nécessité. Mais l'adhésion de l'écriture et de la pensée au sujet : tel est pour lui le critère.

Quand il publie ses premières études littéraires, à Québec, le jeune abbé Roy se trouve, dans le domaine des lettres, devant une situation par beaucoup d'aspects semblable à celle que nous décrivions tout à l'heure. Le romantisme de Crémazie et de Fréchette agonise. Une nouvelle génération prend la relève.

Le romantisme au Canada, que représente-til ? D'abord, les premiers cris articulés de notre peuple dans l'ordre littéraire. Ensuite, très tôt, l'image de notre protestation. Pour la première fois, dans l'ordre littéraire toujours, notre destin tragique prend forme. L'abbé Groulx a dit que nous étions un peuple tragique, puisque par notre condition même, nous vacillions constamment entre l'être et le néant. Les romantiques canadiens ont, avant la lettre, repris cette formule tragique. Mais ils ne l'ont pas reprise que sur le mode littéraire. Le tragique reposait aussi chez eux sur un débat intérieur : celui de la foi, d'abord, celui de l'expression ensuite. A des poètes comme Crémazie et Fréchette, pour ne citer que ceux qui peut-être survivront, le Canada français, tenu pour des raisons historiques à adhérer à des formes de pensée où le libéralisme de l'esprit ne pouvait trouver sa part, ne représentait pas, à l'intérieur même de la religion, qu'une force d'inertie. Il représentait une force hostile à l'éclosion de la pensée poétique. C'est à l'intérieur de cette conception tragique du monde et d'eux-mêmes qu'il faut les juger. Leurs cris, ce sont les cris d'un peuple pauvre, ignorant - c'est aussi un cri contre l'architecture de notre civilisation.

Avec l'avènement de la bourgeoisie riche, la poésie reflète un autre état d'âme. Elle s'évade, elle veut de nouveau protester, cette fois non contre le conformisme de la pensée, mais contre celui de Mammon. Par ailleurs, la langue s'est affermie. A la poésie didactique par manque de mots et de musique verbale, succède le plaisir des notations subtiles de l'âme.

Dès l'abord, $\mathrm{M}^{\mathrm{gr}}$ Camille Roy se trouve profondément engagé dans ce débat. Il ne pouvait qu'applaudir à la nouveauté de la pensée poétique de l'Ecole de Montréal, que donner son assentiment aux essais de virtuosité verbale. Car tout ce qui tendait à rapprocher la littérature canadienne de son idéal, un idéal de perfection, lui souriait. Par ailleurs, le post-romantisme et le symbolisme, dans nos lettres, représentent autre chose qu'une adhésion formelle à une vague littéraire. Imités des Français, ils ont un sens plus profond. Ils sont l'indice d'une transformation, d'une désintégration de la pensée proprement 
religieuse de notre peuple. Le romantisme avait esquissé la tendance; les poètes de l'époque de $\mathrm{M}^{\mathrm{gr}}$ Roy la confirment. Non plus que la liberté linguistique, mais l'apparition d'une liberté religieuse. Les poètes et les romanciers les plus représentatifs de cet essor exercent une influence, certes; mais ils la subissent aussi. En sorte qu'ils ne sont pas, ne peuvent pas être, que l'éclosion spontanée d'une révolte religieuse qui n'ose pas - qui n'ose pas encore - dire son nom. Ils sont un reflet de la classe montante à laquelle ils appartiennent; ils traduisent ses sentiments, ils s'apprêtent à rejeter les canons habituels.

A cette révolte religieuse et littéraire inconsciente, $\mathbf{M}^{\mathrm{gr}}$ Camille Roy a opposé, inconsciemment peut-être, mais avec vigueur, le veto de son credo. Il n'a jamais abattu pour le plaisir d'abattre. Au contraire, il est permis de parler, à son propos, d'une critique évangélique. Mais il a compris, et c'est la plus profonde signification de son œuvre de critique, que ce n'était là qu'une crise de croissance et que même les velléités de révolte reposaient sur les données de la foi. Une formule rendra cette attitude de son esprit : sa foi ne lui a jamais gâté le goût. Qu'il s'agisse de Paul Morin, ou de Nelligan, ou de Jean-Charles Harvey, il a su reconnaître que les cris de révolte pouvaient avoir un sens dans le contexte canadien. Vous trouverez parfois un manque d'adhésion, un refus de suivre les auteurs sur le terrain des idées; jamais il ne s'est livré à ce que l'on appelle un " abattage». Nous voyons dans cette attitude la seule réponse possible à un chrétien et à un prêtre. De là découlent peut-être les critiques qui lui furent souvent faites d'être un bénisseur, un doux critique. Replacée dans son contexte social et dans la perspective de sa charité de prêtre, il semble bien que cette douceur devient plus compréhensible et prend un sens où le mot de vertu a sa place.

$\mathrm{M}^{\mathrm{gr}}$ Camille Roy aura donc, sur le plan littéraire, témoigné en deux sens : pour la vérité par la charité, pour l'art par un grand besoin de perfection. Il donnait lui-même l'exemple d'une langue pure et personnelle. Il l'exigeait des autres. Le reproche qu'il faisait le plus souvent s'adressait à la mollesse du langage. Il croyait qu'une idée n'est pas, si elle n'est pas exprimée avec soin. A la personne et aux idées d'Arthur Buies, par exemple, il ne pouvait vouer un culte; mais au pur écrivain, à l'artiste, il reconnaissait une grandeur certaine.

Tout Camille Roy est là. Il a compris et vécu le mot « fidélité 》. Fidèle à sa foi, à la civilisation de son peuple, à sa conception, qui n'a jamais varié, du beau littéraire. Le premier, sinon l'un des seuls, il a pris notre littérature au sérieux, comme représentant, à l'intérieur de provinces étroites, un pilier de la civilisation française. Il a eu le sentiment de la mission de l'écrivain, de son rôle dans notre société et il a consacré une grande part de sa vie à donner son plein sens à ce rôle, avec une ardeur réfléchie.

\section{Conclusion}

Nous avons essayé de dégager d'une histoire encore toute proche, les idées-forces, la Weltanschauung de $\mathbf{M}^{\mathrm{gr}}$ Camille Roy. L'œuvre de cet homme illustre, et peu lu, aura contenu quelques-unes des 
pensées les plus justes sur notre destin national et sur la piété littéraire. Peu importe, en somme, que ces idées n'aient pas eu le retentissement créateur qui aurait pu être le leur. Peu nous importe et $\mathbf{M}^{\mathrm{gr}}$ Camille Roy, ennemi de la grandiloquence, aurait aimé n'être objet de ferveur que pour quelques a happy few ». Le monde universitaire lui a donné la consécration officielle. Par leur subtilité même ses idées et sa doctrine ne pouvaient attirer que des esprits en somme marginaux. Il n'en reste pas moins que leur action bénéfique se fait encore sentir. Grâce un peu à lui, la civilisation dont nous sommes issus aura joué un rôle dans l'éclosion et la formation de notre pensée nationale. Dans notre littérature, il a fait germer des idées de saine beauté, la nécessité du travail, une certaine assurance dans l'usage de la langue. N'est-ce pas rien?

Son époque avait besoin d'un Malherbe. Il aura joué ce rôle purificateur. Prêtre de nos lettres, il a voué sa vie à ce que le beau a de plus raisonnable. Et en ceci encore, il nous représente; il a en quelque sorte donné les ailes d'une langue châtiée et d'un esprit subtil à notre conformisme. Son époque ne lui eût pas permis d'aller plus loin et ses goûts ne l'ont jamais entraîné vers le bizarre. Mais cet esprit pur connaissait la nuance et sa sympathie pour Nelligan, ange noir qui devait si tôt replier ses ailes maladives, est un indice de la catholicité et de la charité de son esprit.

Refermons donc les pages de ses livres sur cette pensée de Gandhi : - L'âge m'aura appris à croire que non pas l'action, mais l'esprit accomplit une œuvre éternelle. $\mathrm{M}^{\mathrm{gr}}$ Camille Roy, fruit de l'esprit français, universel et éternel, n'eût pas désavoué cette maxime d'un sage moderne.

Jean Éthier BLaIs.

Professeur à l'Université Carleton, Ottawa, Ont. 